\title{
Radio Recombination Lines as a Tool for Study Dynamics of Planetary Nebulae
}

\author{
Sergei Gulyaev \\ Auckland University of Technology, Private Bag 92006, Auckland 1020, \\ New Zealand
}

\begin{abstract}
An observational program for study dynamics of planetary nebulae with the help of RRL is discussed. The expansion velocities may be found from observations of radio recombination lines in different wavelength. The method is free of the microturbulence problem inherent in standard spectroscopic methods. Set of theoretical models was computed for NGC 7027. Observations of RRL with $n>150$ may allow making choice between models with high and low microturbulence.
\end{abstract}

For planetary nebulae with high emission measure the expansion velocities may be found from multiwavelength observations of radio recombination lines (RRL). The method is based on the comparison of the central velocity $V_{L S R}$ of optically thin $\left(\tau_{L+C}<1\right)$ RRL and $V_{L S R}$ obtained from observations of optically thick $\left(\tau_{L+C}>1\right)$ RRL. For most of planetary and diffuse nebulae, the frequency corresponding to the turnover of the bremsstrahlung (critical frequency $\nu_{c r}$ ) is in the range of radio wavelengths. It was shown that the radial velocity of radio recombination lines with $\nu>\nu_{c r}$ is systematically higher than that of lines with $\nu<\nu_{c r}$ (Gulyaev \& Sorochenko 1985). This difference $\Delta V_{L S R}>0$ was interpreted in terms of expansion of nebulae (see also Berulis \& Ershov 1983).

A high-frequency radio line is formed in the whole volume of nebula, transparent in this frequency, whereas low-frequency line emission comes from the outer (closer to the observer) parts of the opaque nebula. In the former case the optical depth $\tau_{L+C}<1$, so the radial velocity of radio lines is the result of averaging (integration) over the whole volume of the nebula. For a symmetrical shape nebula, $V_{L S R}$ is the radial velocity of its geometric centre. In the later case $\tau_{L+C}>1$, and $V_{L S R}$ is determined by the dynamics of the outer (closer to the observer) parts of the nebula. In a simplistic case of a spherical shell and homogeneous expansion

$$
V_{\text {expansion }}=\frac{3}{2}\left(V_{L S R}(\tau<1)-V_{L S R}(\tau>1)\right)=\frac{3}{2} \Delta V_{L S R}
$$

Set of theoretical models was computed for NGC 7027 (Figure 1). We have employed the model of Viner et.al. (1979), using the input parameters from Masson (1989). Provided emission measure EM $=2 \times 10^{6} \mathrm{pc} \mathrm{cm}^{-6}$ (Roelfsema et.al. 1991) the optical depth at $1.5 \mathrm{GHz}$ at the centre of NGC 7027 is $\tau_{C} \approx 2$. As it is seen from Figure 1, observations of RRL with $n \geq 150$ may allow making choice between models with high and low expansion velocities (low and high microturbulence, respectively). Free of the microturbulence problem inherent in standard spectroscopic methods for the expansion velocity measurements (e.g. 


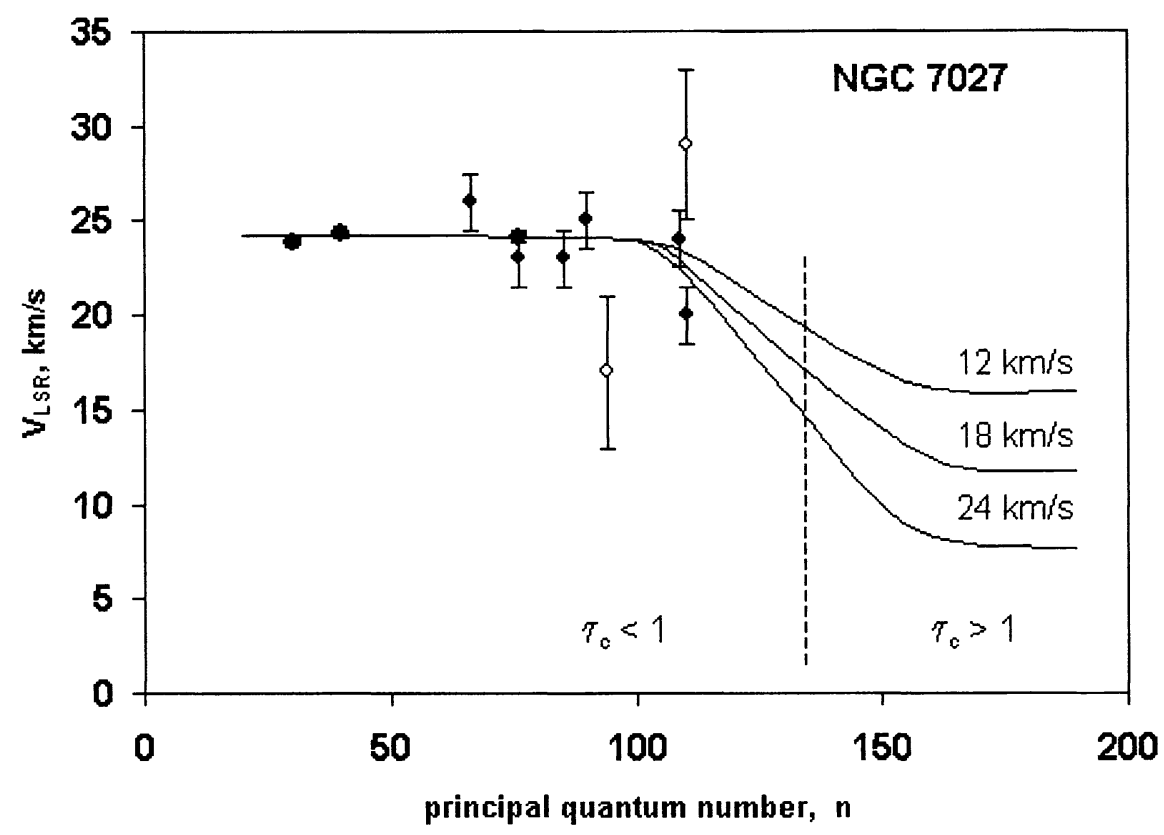

Figure 1. Shows theoretical and measured central velocities $V_{L S R}$ as a function of the principal quantum number $n$ for the planetary nebula NGC 7027. The model of Viner et.al. (1979) with the input parameters from Masson (1989) was employed. Theoretical curves were computed for three values of the expansion velocity: 12,18 , and $24 \mathrm{~km} / \mathrm{s}$

Vallee et.al. 1990), this method can be applied to planetary nebulae with high emission measure.

\section{References}

Berulis, I. \& Ershov, A.1983, Soviet Astronomy Letters, 9, 656

Gulyaev, S. \& Sorochenko, R. 1985, Bull. Abastum. Astrophys. Obs., 59, 135

Masson, C. 1989, ApJ, 336, 294

Roelfsema, P., Goss, W., Pottash, S., \& Zijlstra, A. 1991, A\&A, 251, 611

Vallee, J., Guilloteau, S., Forveille, T. \& Omont, A. 1990, A\&A, 230, 457

Viner, M., Vallee, J., \& Hughes, V. 1979, ApJS, 39, 405 